\title{
CHARGE STABILITY IN LPCVD SILICON NITRIDE FOR SURFACE PASSIVATION OF SILICON SOLAR CELLS
}

\author{
Yongling Ren, Natalita M Nursam, Da Wang and Klaus J Weber \\ Centre for Sustainable Energy Systems, College of Engineering and Computer Science, The Australian National \\ University, Canberra, ACT 0200, Australia
}

\begin{abstract}
Negatively charged dielectric films are of great interest for application to silicon solar cells, since such films offer the possibility of excellent passivation of $p$ type surfaces. This paper investigates the distribution and stability of negative charge in silicon dioxide / LPCVD silicon nitride films which have been negatively charged through the injection of electrons from the silicon substrate. High densities of negative charge $\left(5-10 \times 10^{12} \mathrm{~cm}^{-2}\right)$ can be stored in the silicon nitride films. The negative charge is determined to be mainly concentrated at the oxide/nitride interface. The thermal stability of the charge is shown to be improved by the presence of a barrier layer on top of the silicon nitride. Modelling indicates that the thermal stability is likely to be sufficient for application to solar cells, which will operate at elevated temperatures for 25 years or more.
\end{abstract}

\section{INTRODUCTION}

Currently, surface passivation of crystalline silicon (c-Si) is becoming increasingly important for high-efficiency and low-cost solar cells. The importance of surface passivation becomes greater as the substrate thickness for solar cells is reduced for the sake of material cost reductions. Electrostatic charge can substantially reduce the recombination of excess carriers at semiconductor surfaces, by reducing the concentration of one type of carrier. In principle, either inversion or accumulation conditions (corresponding, for $\mathrm{p}$ type $\mathrm{Si}$, to a large concentration of electrons or holes, respectively) can result in low interface recombination. In practice, however, accumulation conditions are preferred due to the absence of space charge region recombination, the avoidance of parasitic shunts and the fact that, particularly for heavily doped or diffused surfaces, accumulation conditions are much easier to achieve than inversion conditions. [1]

For the passivation of the rear side of conventional cells based on $p$ type material, a dielectric containing negative charge is therefore desirable. Hoex et al $[2,3]$ showed that plasma assisted atomic layer deposited (ALD) $\mathrm{Al}_{2} \mathrm{O}_{3}$ layers can provide excellent surface passivation. The disadvantage is that ALD is a slow process, making the industrial applicability of the process uncertain. We have recently demonstrated that negatively charged silicon nitride layers can lead to a significant improvement in surface recombination at lightly doped or diffused, $p$ type surfaces [4]. Possible structures for charge injection are shown in Fig. 1. They consist of a tunnel oxide, a silicon nitride layer and optionally, a barrier oxide or other dielectric material. As-deposited silicon nitride films are usually positively charged. The process for creating negatively charged nitride over large areas for PV applications is relatively straightforward and amenable to implementation in a production setting. It involves the application of positive charge (using a corona discharge apparatus) on the surface of the silicon nitride film, in order to create an electric field that is sufficiently high to result in the tunnelling of electrons from the silicon into the nitride film. The positive surface charge is subsequently removed.

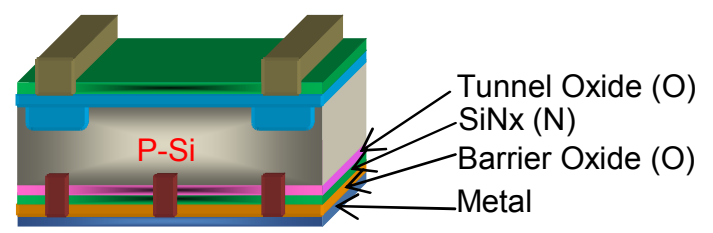

(a)

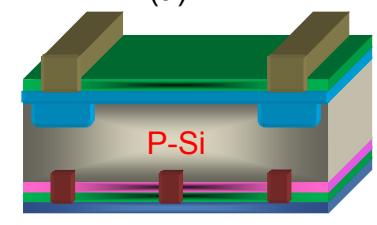

(b)

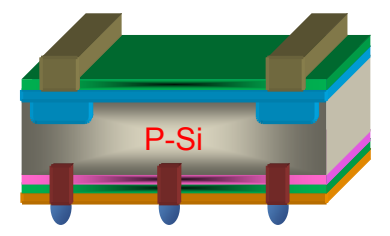

(c)
Figure 1: Possible rear structures incorporating negatively charged nitride layers. (a) and (b): point contacts with overlying sheet metal and with (a) or without (b) an additional barrier layer; (c): grid contact structure.

The dominant defect in near stoichiometric silicon nitride $\left(\mathrm{SiN}_{\mathrm{x}}\right)$ films is known to be the $\mathrm{K}$ centre, consisting of a $\mathrm{Si}$ atom backbonded to three $\mathrm{N}$ atoms. The $\mathrm{K}$ centre displays neutral and charged states. Following the tunneling of electrons from the Si substrate into the nitride film, the electrons can be trapped by the positively charged or neutral $\mathrm{K}$ centres, resulting in a net negative charge in the $\mathrm{SiN}_{\mathrm{x}}$ film. The manipulation of the $\mathrm{SiN}_{\mathrm{x}}$ charge state by charge injection has been exploited for non-volatile memory devices (NVMs). [5,6] In this paper, we investigate the stability of silicon dioxide/silicon nitride stacks in more detail, in order to determine under what conditions the charge is sufficiently stable for photovoltaic applications.

\section{EXPERIMENTAL DETAILS}

Samples used for $\mathrm{C}-\mathrm{V}$ measurements were $\mathrm{Cz}$ p-type, 1$10 \Omega$-cm (100) wafers. After a standard RCA clean, an 
oxide layer around $20 \mathrm{~nm}$ thick was thermally grown at $900^{\circ} \mathrm{C}$. $\sim 100 \mathrm{~nm}$ stoichiometric $\mathrm{Si}_{3} \mathrm{~N}_{4}$ was deposited using low pressure chemical vapor deposition at $775^{\circ} \mathrm{C}$ and 0.45 torr, with an ammonia to dichlorosilane (DCS) flow ratio of 4:1. On some samples, $\sim 40 \mathrm{~nm} \mathrm{PECVD} \mathrm{SiO}_{2}$ was subsequently deposited, resulting in either silicon-oxidenitride (SON) or silicon-oxide-nitride-oxide (SONO) structures. Prior to measurement, all insulator layers were removed from the rear of the samples, and $\sim 80 \mathrm{~nm}$ Al dots with an area of $\sim 0.0039 \mathrm{~cm}^{2}$ were evaporated on the front. $\mathrm{In} / \mathrm{Ga}$ contacts were formed on the rear of the samples. In order to inject electrons into the silicon nitride film, progressively increasing positive bias voltages $\mathrm{V}_{\text {app }}$ were applied to the front metal contacts for periods of several seconds. Following each bias voltage, a high frequency $\mathrm{C}$ $\checkmark$ sweep was carried out to determine the flatband voltage of the sample. The use of bias voltages for charge injection rather than corona charging allows the charging and charge decay mechanisms to be investigated in more detail. Following charge injection, selected samples were stored at elevated temperatures for extended periods, to assess the charge stability in the structure.

\section{RESULTS AND DISCUSSION}

\section{Charge distribution}

In order to determine the location of injected charge in LPCVD $\mathrm{Si}_{3} \mathrm{~N}_{4}$ layers, a nitride-oxide-Si (SON) structure consisting of a $20 \mathrm{~nm}$ thick thermal $\mathrm{SiO}_{2}$ layer and a $50 \mathrm{~nm}$ thick layer of LPCVD $\mathrm{Si}_{3} \mathrm{~N}_{4}$ was used. Following the injection of electrons into the nitride film, the flatband voltage $\left(\mathrm{V}_{\mathrm{fb}}\right)$ was measured as the $\mathrm{Si}_{3} \mathrm{~N}_{4}$ film was progressively removed by etching. Fig. 2 shows the resulting curve of $V_{\mathrm{fb}}$ as a function of remaining nitride layer thickness. $V_{\mathrm{fb}}$ initially decreases linearly with decreasing nitride layer thickness and then becomes nearly constant for a thickness of around $6 \mathrm{~nm}$. This indicates that the charge is mainly concentrated near the nitride/oxide interface, which is consistent with previous reports [7]. However, the etchback method can only give a very approximate indication of the location of the charge.

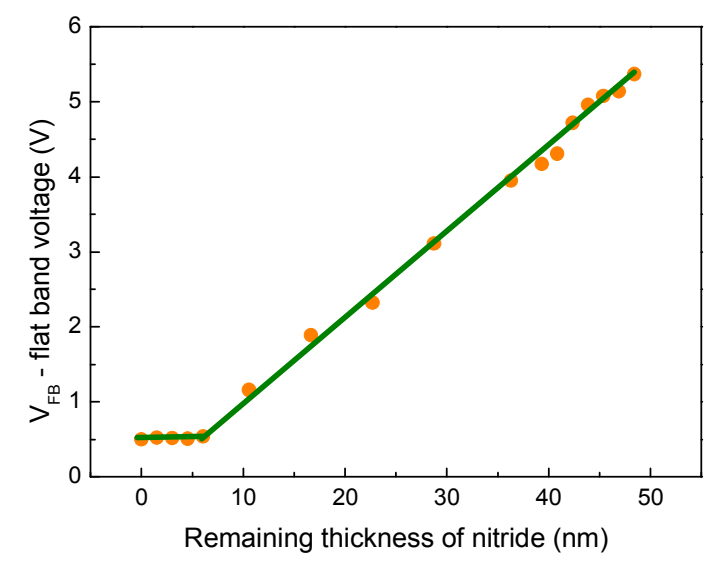

Figure 2 the $V_{\mathrm{fb}}$ versus remaining nitride thickness

\section{Charge injection}

Since the charge appears to be located within the first $\sim 10 \mathrm{~nm}$ from silicon oxide-nitride interface, we can apply eq. (1) to estimate the charge density in our nitride films with reasonable accuracy:

$$
\mathrm{N}=\frac{C_{i}}{q A}\left(\varphi_{m s}+V_{f b}\right)
$$

where $\mathrm{N}$ is the fixed charge density in silicon nitride in units of fundamental charges per $\mathrm{cm}^{2}, \mathrm{C}_{\mathrm{i}}$ is the capacitance of nitride, $q$ is the elementary charge, $\varphi_{\mathrm{ms}}$ is the work function difference between the metal and silicon, and $A$ is the Aluminum electrode area. For the samples used, $\varphi_{\mathrm{ms}}$ is $\sim 0.87 \mathrm{~V}[8,9]$.

Fig. 3 shows a typical plot of $V_{\mathrm{fb}}$ as a function of applied bias. The corresponding negative charge density calculated via equation (1) is given as well. It can be seen that a threshold voltage (in this case, $\sim 40 \mathrm{~V}$ ) is required before significant carrier injection occurs. The corresponding electric field strength across the tunnel oxide is $\sim 6 \mathrm{MV} / \mathrm{cm}$. At an applied bias above $\sim 70 \mathrm{~V}, \mathrm{~V}_{\mathrm{fb}}$ saturates, probably indicating that all of the available $\mathrm{K}$ centres have been charged. The saturated negative charge density is $\sim 4.74 \times 10^{12} \mathrm{~cm}^{-2}$ in this case.

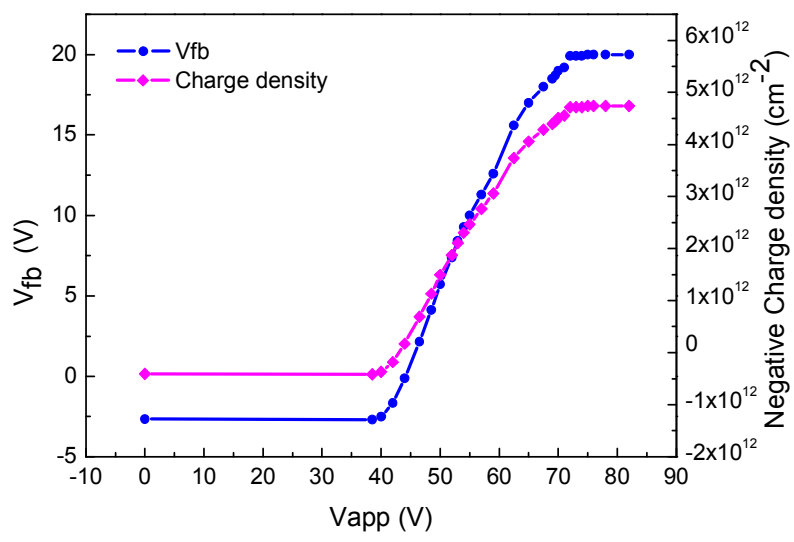

Figure 3 Flat band voltage / negative charge density as the function of applied bias voltage

\section{Charge stability at elevated temperature}

Studies on NVMs[5] have shown that several mechanisms are responsible for charge decay in the nitride. However, the dominant mechanism, particularly at elevated temperatures, consists of the thermal emission of carriers from the negatively charged $\left(\mathrm{K}^{-}\right)$centre to the nitride conduction band, followed by Fowler-Nordheim tunnelling 
across the tunnel oxide. This is illustrated in Fig. 4. (mechanism 1)

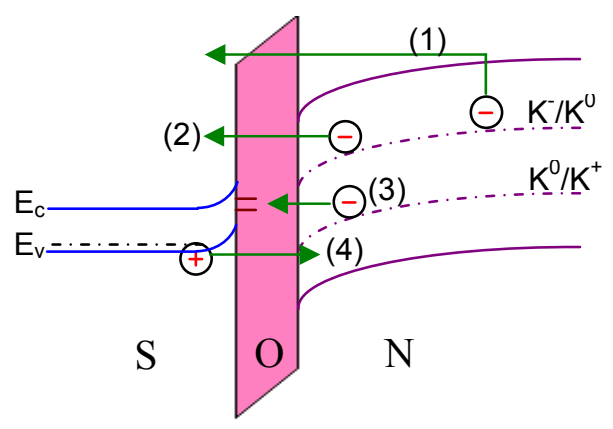

\section{Figure 4 Charge decay mechanisms for the excess electron state}

Following on from the work of McWorther at al. [6] and Kim et al [7] we can model the decay of the charge in the nitride film based on expressions describing the emission of charges from the $\mathrm{K}^{-}$centre to the nitride conduction band, and Fowler-Nordheim (F-N) tunneling across the oxide barrier.

The thermal emission of electrons from a $\mathrm{K}^{-}$centre is given by

$$
e_{t h}=A T^{2} \exp \left(-\frac{E_{K^{-}}}{k T}\right)
$$

where $E_{k}{ }^{-}$is the energy level of $\mathrm{K}$ centres below the nitride conduction band edge, $A$ is a temperature independent constant, $T$ is the temperature in Kelvin, and $k$ is Boltzmann's constant.

The F-N tunneling probability is given by:

$$
\mathrm{P}_{\mathrm{F}-\mathrm{N}}=\operatorname{Kexp}\left[\frac{-4 \sqrt{2 m^{*}}\left(q \phi_{n x}\right)^{3 / 2}}{3 \hbar q E}\right]
$$

Where $q$ is the electronic charge, $E$ is the electric field strength $(\mathrm{V} / \mathrm{cm}), \hbar$ is the reduced Plank constant, $\phi_{n x}$ is the barrier height at the nitride-oxide interface, $m^{*}$ is the average effective mass and $\mathrm{K}$ is a constant.

In order to model the decay of charge, we assume a certain density and energy of initially charged ( $\left.\mathrm{K}^{-}\right)$defects. We assume that carriers, once emitted into the silicon nitride conduction band, are not subsequently captured again by a defect but remain in the conduction band until they tunnel across the oxide and into the silicon substrate. This leads to a density of carriers in the nitride conduction band at time $t$ of $\mathrm{N}_{\mathrm{C}}(\mathrm{t})$. The rate of tunneling of carriers across the oxide barrier (and therefore the rate of charge decay) is then given by

$$
N(t)=N_{C}(t) \cdot P_{F-N}
$$

An important feature of the F-N equation is that the tunneling probability is extremely sensitive to the electric field strength, with a higher electric field strength leading to a dramatically increased tunneling probability.

Fig. 5 shows an example of the modeled charge decay for 3 different temperatures. Some of the key features of the graph are: i) the onset of charge decay occurs after a certain amount of time; ii) the temperature impacts significantly on the time of the onset of the decay; and iii) for sufficiently low charge densities (and therefore electric field strength across the oxide) the charge decay becomes independent of temperature. This latter feature is a result of the strong electric field dependence of the $\mathrm{F}-\mathrm{N}$ tunneling probability.

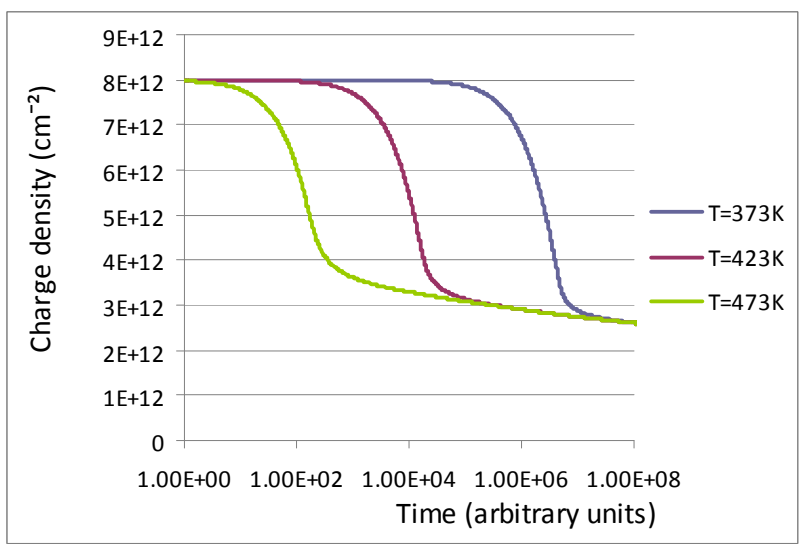

Figure 5: Modelled decay of charge density for different storage temperatures. The single energy level of the $\mathrm{K} / \mathrm{K}$ trap is assumed to be $1.4 \mathrm{eV}$ below the nitride conduction band, and $\phi_{n x}$ is taken to be $1.05 \mathrm{eV}$ [8].

Fig. 6 shows the change in the effective charge density of samples following storage at $150^{\circ} \mathrm{C}$ in atmosphere, for samples with an oxide-nitride (SON) as well as an oxidenitride-oxide (SONO) structure. The charge decay of the SON sample is noticeably faster than that of the SONO sample, indicating the charge loss to the atmosphere can be significant. This mechanism is likely to be significantly reduced in a solar cell embedded in a module. 


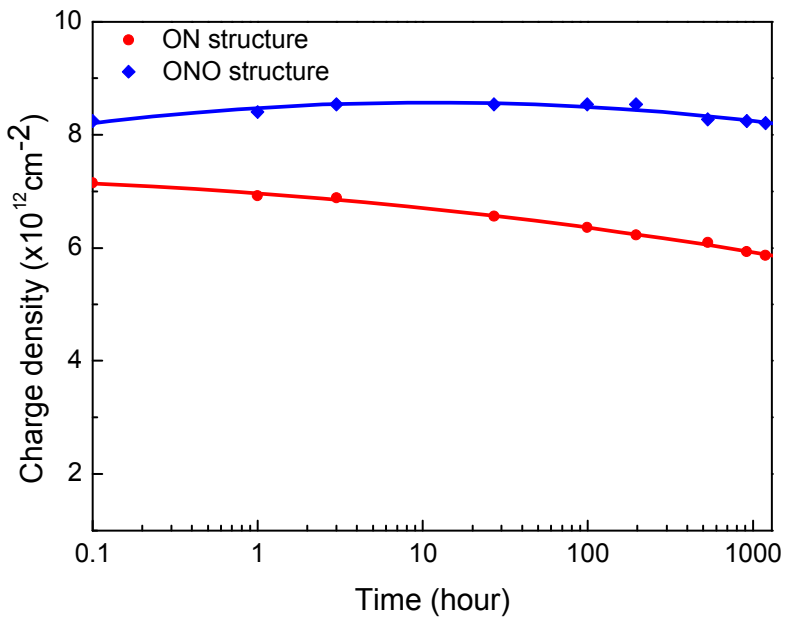

Figure 6: Effective charge density (calculated using eq. (1) as a function of storage time for silicon-oxide nitride (SON) and silicon-oxide-nitride-oxide (SONO) samples at $150^{\circ} \mathrm{C}$.

The SONO sample shows a slight increase in effective charge density, followed by a decrease. The initial increase is a common feature of the samples we have investigated. We believe that this is the result of some rearrangement of the charge within the nitride film with charge moving closer to the oxide-nitride interface. This of course also indicates that at least the initial determination of charge density using eq. (1) is underestimated by $10 \%$ or perhaps even more. Unfortunately, this feature also makes it more difficult to model the experimental results.

Fig. 7 compares the measured charge decay at $150^{\circ} \mathrm{C}$ and $200^{\circ} \mathrm{C}$ for otherwise identical samples with an ONO structure. It also shows the modeled charge decay at $100^{\circ} \mathrm{C}, 150^{\circ} \mathrm{C}$, and $200^{\circ} \mathrm{C}$ respectively. It can be seen that, as expected, a higher temperature leads to an earlier onset of charge decay.

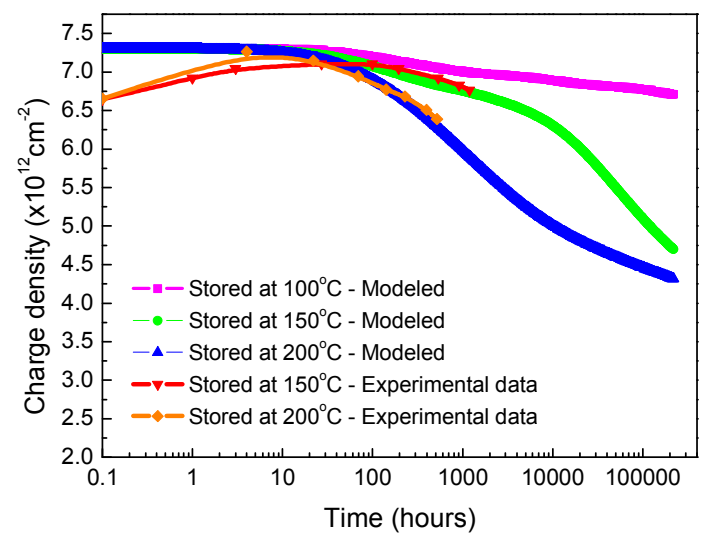

Figure 7: Modeled charge decay at $100^{\circ} \mathrm{C}, 150^{\circ} \mathrm{C}$, and $200^{\circ} \mathrm{C}$ and measured Effective charge density (calculated using eq. (1)) as a function of storage time for SONO samples at 150 and $200^{\circ} \mathrm{C}$

The experimental and modeling results strongly indicate that, at normal module operating temperatures, the charge density in the nitride films will remain at above $5 \times 10^{12} \mathrm{~cm}^{-2}$ for the life of the module. It is generally found [9] that a charge density of $\sim 5 \times 10^{12} \mathrm{~cm}^{-2}$ is sufficient for optimum passivation. However, further work will be required to improve the charge decay model and obtain good agreement with experimental results over a broad range of conditions.

\section{CONCLUSION}

In conclusion, we have demonstrated that high densities of negative charge can be stored in silicon nitride mainly at silicon oxide-silicon nitride interface. The thermal stability of the charge is shown to be improved by the presence of a barrier layer on top of the silicon nitride. Combining experimental and modeling results, it indicates that charge in silicon oxide/LPCVD nitride stacks is sufficiently thermally stable for PV applications. With lower temperature, the charge decay is slower. However, even at elevated temperature $150^{\circ} \mathrm{C}$, the charge density is still enough for the optimum surface passivation after around 25years. Thus, negatively charged silicon nitride films could be used for certain applications in solar cells.

\section{ACKNOWLEDGEMENT}

The authors would like to thank Mr. Bruce Condon and Ms. Nina De Caritat for the assistance on maintaining the $\mathrm{C}-\mathrm{V}$ equipment and chemical processing. Financial support by the Australian Research Council and the Australian National University $\mathrm{PhD}$ scholarship is gratefully acknowledged.

\section{REFERENCES}

[1] S.Dauwe, L. Mittelstadt, A. Metz, and R. Hezel, "Experimental Evidence of Parasitic Shunting in Silicon Nitride Rear Surface Passivated Solar Cells", Progress in Photovoltaics, 2002, pp. 271-278.

[2] B. Hoex et al., "Excellent passivation of highly doped $p$ type $\mathrm{Si}$ surfaces by the negative-charge-dielectric $\mathrm{Al}_{2} \mathrm{O}_{3}$ " Appl. Phys. Lett. 91, 2007, pp. 112107-1-112107-3.

[3] B. Hoex et al., "Ultralow surface recombination of $c-\mathrm{Si}$ substrates passivated by plasma-assisted atomic layer deposited $\mathrm{Al}_{2} \mathrm{O}_{3}$ ", Appl. Phys. Lett. 89, 2006, pp. 0421121-042112-3.

[4] K. J. Weber et al., "Improved silicon surface passivation achieved by negatively charged silicon nitride films" Appl. Phys. Lett. 94, 2009, pp. 063509-1-063509-3.

[5] M. H. White, Y. L. Yang, A. Purwar, and M. L. French, "A Low Voltage Sonos Nonvolatile Semiconductor Memory 
Technology," 1996 Int'l Nonvolatile Memory Technology Conference, 1996, pp. 52-57.

[6] P.J. McWhorter, S.L. Miller and T.A. Dellin, "Modeling the memory retention characteristics of silicon-nitrideoxide-silicon nonvolatile transistors in a varying thermal environment". J. Appl. Phys. 68, 1990, pp. 1902-1909.

[7] T. H. Kim, J. S. Sim, J. D. Lee, H. C. Shin, and B. Park, "Charge decay characteristics of silicon-oxide-nitrideoxide-silicon structure at elevated temperatures and extraction of the nitride trap density distribution" Appl. Phys. Lett. 85, 2004, pp. 660-662.

[8] Jang Uk. Lee et al., "Optical capacitance-voltage characterization of charge traps in the trapping nitride layer of charge trapped flash memory devices" Appl. Phys. Lett. 2007, 91, pp. 223511-1 - 223511-3.

[9] K. J. Weber, H. Jin, C. Zhang, N. Nursam, W. E. Jellett and K. R. McIntosh, "SURFACE PASSIVATION USING DIELECTRIC FILMS: HOW MUCH CHARGE IS ENOUGH?" 24th European Photovoltaic Solar Energy Conference, 21-25 September 2009, Hamburg, Germany, pp. 534-537. 